\title{
Coronary Artery Calcium Scoring by Cardiac CT as A Screening Tool in 40-45 Years Age Group - Predictor of Future Risk for Cardiovascular Events- Systemic Review
}

\author{
Kumar S, Sharma S
}

Department of Cardiac Radiology, AIIMS, New Delhi, India

\section{Background}

Atherosclerosis is a systemic process that can develop as early as the second or third decade of life. A significant percentage of patients who experience acute coronary syndromes often have non-obstructive coronary artery disease and thus cannot be diagnosed by their symptoms or conventional functional stress testing. It has been proposed that early detection of atherosclerosis would generate novel opportunities for primary prevention through changes in lifestyle or even through drug therapy, especially in patients at high cardiovascular risk. Calcium scoring is a simple, reproducible, and widely available test that has been extensively validated over the past two decades. Most incidence of coronary disease occurs in previously asymptomatic individuals who were considered to be at a lower risk by traditional screening methods. There is a definite advantage if these individuals could be reclassified into a higher risk category, thereby impacting disease outcomes favorably. Coronary artery calcium scores have been recognized as an independent

Corrospondence to: Dr. Sanjiv Sharma, Professor and Head, Department of cardiac Radiology, AIIMS, New Delhi-110029, India.

E-mail: meetisv@yahoo.com marker for adverse prognosis in coronary disease. Multiple population based studies have acknowledged the shortcomings of risk prediction models such as the Framingham risk score or the Procam score.

\section{Risk Factor for Coronary Artery Disease}

In no other disease entity is the assessment of risk factors more important, than in CAD. This is due to cost implications and also due to the fact that events, sometimes fatal, can be averted. Risk stratification in CAD assumes greater importance also because it determines the type of tests that may be ordered by a clinician and also helps him or her choose between invasive and noninvasive treatment modalities. ${ }^{1}$ The Framingham risk score was based on data from the Framingham Heart Study, one of the longest running prospective studies on CAD, and estimates 10-year coronary heart disease outcomes. It includes age, sex, total cholesterol, HDL cholesterol, smoking status, systolic blood pressure and use of anti-hypertensives. $^{2}$ It has long been acknowledged that these so called "traditional risk factors" are unable to account for all cases of CAD. It is also evident that the findings from the Framingham cohort cannot be generalized to the rest of the population or other ethnic groups, as that was a study done on an almost entirely Caucasian population. This 
has spurred interest in the quest for novel cardiovascular risk factors. Examples include, CRP, Lipoprotein-A, Homocysteine, abdominal obesity, coronary artery calcium, carotid intima media thickness, lack of physical activity and psychosocial stress to name just a few.

\section{The Role of Calcium in the Athero- sclerotic Plaque}

It will be useful to recapitulate the evolution of the atherosclerotic plaque and the role of calcium at a microscopic and macroscopic level in it. Endothelial cells, leukocytes and intimal smooth muscle cells are the main cellular agents responsible for atherosclerosis. Exposure of vascular endothelium to atherogenic stimuli results in extra cellular lipid accumulation which leads to recruitment of leukocytes. The major leukocytes involved in this process are monocytes and T-Lymphocytes. The monocytes subsequently imbibe lipid and evolve into the "foam cell" or lipid laden macrophage which is considered the pathological hallmark of the atherosclerotic plaque. CAC occurs, and increases with age and develops in advanced atherosclerotic plaques although it is worth noting that absence of calcification does not imply absence of atherosclerosis. ${ }^{4}$ The noncalcified atherosclerotic lesions (NCALs) or "soft plaques" occupy a very prominent role in CAD as they are thought to be more unstable and therefore more "vulnerable". However, CAC is not specific for luminal obstruction as there are other factors that come into play including vascular remodeling which impact arterial lumen and coronary blood flow. Arterial lumen was studied in humans and nonhuman primates by Clarkson, et al. who concluded that lack of remodeling may be a major factor in certain individuals with coronary atherosclerosis not developing complications such as myocardial infarction. ${ }^{5}$ Atherosclerotic calcification mainly involves the intimal layer of the artery as opposed to the more classical medial calcification (Monckeberg's medial calcinosis), which is common in diabetic patients. ${ }^{6}$ Numerous bone proteins are found in the atherosclerotic plaque and Osteopontin, a protein involved in mineralization has been specifically associated with calcific coronary atheroma and is thought to play an important role in the onset and progression of disease in humans. ${ }^{7}$ Smooth muscle cells are thought to secrete cytokines that facilitate calcium formation in the atheroma. In vitro studies have been performed, in which the ionic concentrations of calcium and phosphorous were simulated to mimic serum concentrations, and the resulting carbonate bioapatite precipitates were found to resemble closely, the calcified atherosclerotic plaques seen in human blood vessels. $^{8}$

\section{Calcium Scores}

The amount of calcium in the coronary arteries can be quantitated. Various methods have been proposed for this purpose. The most attractive and the most commonly used is the Agatston score. Other methods described include calcium volume and mass (mineral) scores.

\section{Agatston Scores}

Agatston, et al. described a novel way of measuring coronary artery calcium in $1990 .{ }^{9}$ They used ultra fast CT to measure total calcium scores based on the number, areas 
and peak Hounsfield computed tomographic numbers of the calcific lesions detected. Subsequent studies have confirmed the high correlation between calcium scores and histopathologic coronary disease and also that absence of calcification was highly indicative of absence of CAD. ${ }^{10,11}$ Inter reader variability of the Agatston score is about $3 \%$, intra reader variability is less than $1 \%$ and inter scan variability is thought to be about $15 \% .{ }^{9}$ The lower the score, the lower your risk of coronary heart disease. There are four categories of scores; less than 10, meaning minimal to no calcium was found, and those with scores of 11 to 99 who have a moderate amount of calcification. A score between 100 and 399 is classified as increased calcification and any score over 400 signifies extensive calcium deposits. According to the American Heart Association, if the Agatston score is over 1,000 , there is 20 percent chance of having a serious or fatal cardiac episode within one year of testing.

The actual calcium score, although an indicator of overall disease extent, may not be as useful for predicting coronary events as its percentile ranking. Recent studies have emphasized that a calcium score above the 75th percentile for age and sex may increase heart risk an order of magnitude above that for individuals with scores below the 25th percentile. Thus, even small scores that are much higher than those anticipated for age and sex may be better predictors of risk. For instance, a calcium score of 40 in a 40 -yearold man would place him well above the 95th percentile and engender a risk of a cardiac event during the next 3-6 years that may well exceed that of a 70-year-old with a similar score, who would rank for that age group below the 10th percentile. To further stratify risk the score should be compared with age specific score database developed by Hoff et al.

\section{Calcium Score in Predicting the Risk of cardiac Events}

Coronary calcium does predict risk since, in some measure it quantifies the total atherosclerotic burden. ${ }^{14} \mathrm{~A}$ majority of acute cardiovascular events, i.e., strokes and myocardial infarctions occur in subjects who are not considered to be at high risk for these events by traditional screening techniques in vogue, for e.g., the Framingham Risk Score or Procam score. Furthermore, calcification is known to be intimately associated with atherosclerosis even from a preatheroma stage and is known to increase with age. ${ }^{15,16}$ Large observational cohorts studied to date, with zero calcium scores are known to have a low adverse cardiac event risk. ${ }^{17}$ An American Heart Association writing group stated that a CAC score of zero ( $\mathrm{CAC}=0$; ie, no calcified plaque detected) indicated :

1) that the presence of atherosclerotic plaque, including unstable or vulnerable plaque, was highly unlikely;

2) that the presence of significant luminal obstructive disease was highly unlikely (negative predictive value on the order of $95 \%$ to $99 \%$ ); and

3 ) that the risk of a cardiovascular event in the next 2 to 5 years was quite low ( 0.1 per 100 person-years).

In addition, positive calcium score and incremental values are associated with higher sensitivity for atherosclerotic diseases, increased risk for future cardiovascular events however lower specificity for 
coronary obstructive diseases. A large observational cohort of over 10,377 asymptomatic subjects was followed in a study by Shaw et al. and with their risk adjusted model, the relative risk of mortality was $1.64,1.74,2.54$, and 4.03 times greater for calcium scores of 11-100, 101-400, 4011,000 , and greater than 1,000 , respectively, compared with the relative risk for a calcium score of 10 or less. A large observational cohort of over 25,000 asymptomatic subjects was followed in a study by Budoff et al. in which coronary artery calcification (CAC) was found to be an independent predictor for all cause mortality. ${ }^{18}$ There is a wealth of data which supports the use of CAC as a surrogate for coronary atherosclerosis confirmed by correlation of angiographic, histopathologic and necropsy. ${ }^{19,20}$ It was shown by Mautner et al. that the amount of calcium deposits measured by EBCT correlates very well with histomorphometric measurements. ${ }^{21}$ A study by Rumberger et al. demonstrated that increasing amounts of coronary artery calcium by $\mathrm{CT}$ were found to correlate with more advanced atherosclerotic involvement of the coronaries detected by histological section. $^{22}$

Agatston score define the degree of atherosclerosis in coronary artery and classify the patients in low, intermediate and high risk group for future cardiovascular event as a separate marker and helps in planning management whether to adapt aggressive or non aggressive approach. It is proposed that early detection of atherosclerosis in 40-45 years age group will classify these patients in low, intermediate and high risk groups for future cardiovascular events apart from other conventional risk factors and will initiate early and adequate timely management for prevention of future events.

\section{References}

1. Litchfield RL. Noninvasive tests for cardiac risk stratification. Which ones are most prognostic? Postgrad Med 2004;115:30-6.

2. Wilson PWF, D'Agostino RB, Levy D, Belanger AM, SilbershatzH, Kannel WB. Prediction of coronary heart disease using risk factor categories. Circulation 1998;97:1837-47.

3. Michos ED, Nasir K, Braunstein JB. Framingham risk equationunderestimates subclinical atherosclerosis risk in asymptomatic women. Atherosclerosis 2006;184:201-6.

4. Vashist A, Bonow RO. Screening for coronary atherosclerosis withcoronary calcium scoring: The "shape" of things to come? J Nucl Cardiol 2008;15:162-9.

5. Clarkson TB, Prichard RW, Morgan TM, Petrick GS, Klein KP. Remodeling of coronary arteries in human and nonhuman primates. JAMA 1994;271: 289-94.

6. Demer LL. Vascular calcification and osteoporosis: inflammatory responses to oxidized lipids. Int J Epidemiol 2002;31: 737-41.

7. Fitzpatrick LA, Severson A, Edwards WD, Ingram RT. Diffuse calcification in human coronary arteries. Association of osteopontin with atherosclerosis. J Clin Invest 1994; 94:1597-604. 
8. Olsson, L.-F, Sandin K, Odselius R, Kloo L. In vitro formation of nanocrystalline carbonate apatite - a structural and morphologicalanalogue of atherosclerotic plaques. Eur J Inorg Chem 2007;4123-7.

9. Agatston AS, Janowitz WR, Hildner FJ, Zusmer NR, Viamonte M, Jr., Detrano R. Quantification of coronary artery calcium using ultrafast computed tomography. J Am Coll Cardiol 1990;15:827-32.

10. Simons DB, Schwartz RS, Edwards WD, Sheedy PF, Breen JF, Rumberger JA. Noninvasive definition of anatomic coronary artery disease by ultrafast computed tomographic scanning: a quantitative pathologic comparison study. J Am Coll Cardiol 1992;20:111826.

11. Rumberger JA, Simons DB, Fitzpatrick LA, Sheedy PF, Schwartz RS. Coronary artery calcium area by electron-beam computed tomography and coronary atherosclerotic plaque area: a histopathologic correlative study. Circulation 1995;92:2157-62.

12. Callister TQ, Cooil B, Raya SP, Lippolis NJ, Russo DJ, Raggi P. Coronary artery disease: improved reproducibility of calcium scoring with an electron-beam CT volumetric method. Radiology 1998; 208:807-14.

13. Moselewski F, O'Donnell CJ, Achenbach S. Calcium concentration of individual coronary calcified plaques as measured by multidetector row computed tomography. Circulation 2005;111: 3236-41
14. Weintraub WS, Diamond GA. Predicting cardiovascular events with coronary calcium scoring. N Engl J Med 2008; 358: 1394-6.

15. Roijers RB, Dutta RK, Cleutjens JP, Mutsaers PH, de Goeij JJ, van der Vusse GJ. Early calcifications in human coronary arteries as determined with a proton microprobe. Anal Chem 2008;80: $55-61$.

16. Hoffmann U, Brady TJ, Muller J. Cardiology patient page. Use of new imaging techniques to screen for coronary artery disease. Circulation 2003;108:e50-3.

17. Shareghi S, Ahmadi N, Young E, Gopal A, Liu ST, Budoff MJ. Prognostic significance of zero coronary calcium scores on cardiac computed tomography. J Cardiovasc Comput Tomogr 2007; 1:155-9.

18. Budoff MJ, Shaw LJ, Liu ST, et al. Long-term prognosis associated with coronary calcification: observations from a registry of 25,253 patients. J Am Coll Cardiol 2007;49:1860-70.

19. Rifkin RD, Parisi AF, Folland E. Coronary calcification in the diagnosis of coronary artery disease. Am J Cardiol 1979;44:141-7.

20. Rumberger JA, Sheedy PF 3rd, Breen JF, Schwartz RS. Coronary calcium, as determined by electron beam computed tomography, and coronary disease on arteriogram : effect of patient's sex on diagnosis. Circulation 1995; 91: 1363-7. 
21. Mautner GC, Mautner SL, Froehlich J. Coronary artery calcification: assessment with electron beam CT and histomorphometric correlation. Radiology 1994;192:619-23.

22. Rumberger JA, Schwartz RS, Simons DB, Sheedy PF 3rd, Edwards WD, Fitzpatrick LA. Relation of coronary calcium determined by electron beam computed tomography and lumen narrowing determined by autopsy. Am J Cardiol 1994;73:1169-73. 